\title{
Controversies in the management of clinical stage 1 testis cancer
}

Gregory J. Nason ${ }^{1}$; Peter Chung ${ }^{2}$; Padraig Warde ${ }^{2}$; Robert Huddart ${ }^{3}$; Peter Albers ${ }^{4}$; Christian Kollmannsberger ${ }^{5}$; Christopher M. Booth ${ }^{6}$; Aaron R. Hansen ${ }^{7}$; Philippe L. Bedard ${ }^{7}$; Lawrence Einhorn ${ }^{8}$; Craig Nichols ${ }^{9}$; Ricardo A. Rendon ${ }^{10}$; Lori Wood $^{11}$; Michael A.S. Jewett $^{1}$; Robert J. Hamilton ${ }^{1}$

${ }^{1}$ Division of Urology, Department of Surgery, Princess Margaret Cancer Centre, Toronto, ON, Canada; ${ }^{2}$ Department of Radiation Oncology, Radiation Medicine Program, Princess Margaret Hospital, University of Toronto, Toronto, ON, Canada; ${ }^{3}$ The Institute of Cancer Research and the Royal Marsden NHS Foundation Trust, Sutton, United Kingdom; ${ }^{4}$ Department of Urology, Heinrich-Heine University, Medical Faculty, Düsseldorf, Germany; ${ }^{5}$ British Columbia Cancer Agency Vancouver Cancer Centre, University of British Columbia, Vancouver, BC, Canada; ${ }^{6}$ Cancer Care and Epidemiology, Cancer Research Institute, Queen's University, Kingston, ON, Canada; ${ }^{7}$ Division of Medical Oncology and Hematology, Princess Margaret Cancer Centre, Toronto, ON, Canada; ${ }^{8}$ Department of Oncology, Indiana University School of Medicine, Indianapolis, IN, United States; ${ }^{9}$ Testicular Cancer Multidisciplinary Clinic, Virginia Mason Medical Center, Seattle, WA, United States; ${ }^{10}$ Department of Urology, Dalhousie University, Halifax, NS, Canada; ${ }^{11}$ Division of Medical Oncology, Queen Elizabeth II Health Sciences Centre, Halifax, NS, Canada

Summary of the Canadian Testis Cancer Workshop (The Westin Toronto Airport Hotel, Toronto, ON, Canada, November 15-17, 2018)

Group of Multidisciplinary Experts: Uro-Oncology, Medical Oncology, Radiation Oncology

Cite as: Can Urol Assoc J 2020 June 9; Epub ahead of print. http://dx.doi.org/10.5489/cuaj.6722

Published online June 9, 2020

$* * *$

\section{Abstract}

In November 2018, The Canadian Testis Cancer Workshop was convened. The two-day workshop involved urologists, medical and radiation oncologists, pathologists, radiologists, physician's assistants, residents and fellows, nurses, patients and patient advocacy groups. One of the goals of the workshop was to discuss the challenging areas of testis cancer care where guidelines may not be specific. The objective was to distill through discussion around cases, expert approach to working through these challenges. Herein we present a summary of discussion from the workshop around controversies in the management of clinical stage 1 (CS1) disease. CS1 represents organ confined non-metastatic testis cancer that represents approximately $70-80 \%$ of men at presentation. Regardless of management, CS1 has an excellent prognosis. However, without adjuvant treatment, approximately $30 \%$ of CS1 nonseminomatous germ cell tumors (NSGCT) and 15\% of CS1 seminoma relapse. The workshop reviewed that while surveillance has become the standard for the majority of patients with CS1 disease there remains debate in the management of patients at high-risk of 
relapse. The controversy in the management of CS1 testis cancer surrounds the optimal balance between the morbidity of overtreatment and the identification of patients who may derive most benefit from adjuvant treatment. The challenge lies in a shared decision process where discussion of options extends beyond the simple risk of relapse but to include the longterm toxicities of adjuvant treatments and the favorable cancer-specific survival.

\section{Introduction}

Testis cancer is the most common malignancy in men aged 15-29 years. There are approximately 1100 new cases diagnosed in Canada per year and 70-80\% are clinical stage 1 (CS1) at diagnosis [1]. CS1 represents organ confined marker negative disease and has an excellent prognosis, with cancer-specific survival of $99 \%$, regardless of management choice. Without adjuvant treatment, approximately $30 \%$ of CS1 nonseminomatous germ cell tumors (NSGCT) and approximately $15 \%$ of CS1 seminoma relapse [2,3]. While surveillance has become standard for the majority of patients with CS1 disease there remains debate over management of patients with high-risk characteristics for relapse [4,5].

Adjuvant therapies clearly reduce the risk of relapse. However, a shared decisionmaking process with patients should delve beyond relapse risk to include the potential longterm toxicities of adjuvant therapy and the equivalent cancer-specific survival $[6,7]$. Patients have difficulties weighing complex information regarding multiple outcomes that are important to them and the lack of level 1 evidence to direct a patient towards their optimal treatment option leads to more discrepancies.

In November 2018, The Canadian Testis Cancer Workshop was convened in Toronto. This two-day meeting was comprised of urologists, medical and radiation oncologists, pathologists, radiologists, physician's assistants, residents and fellows, nurses, patients and patient advocacy groups - all with an interest in testis cancer. One of the goals of the workshop was to discuss the challenging areas of testis cancer care where there are no universally accepted standard. The objective was to distill, through discussion around cases, expert approach to working through these challenges.

Herein we present a summary of discussion from the workshop around controversies in the management of CS1.

\section{Overview of management options for CS1}

Treatment options for CS1 seminoma include active surveillance, para-aortic $+/$ - pelvis radiation or chemotherapy (typically carboplatin $\mathrm{x} 1$ or 2 cycles). The EAU guidelines advise offering surveillance 'if the facilities are available and the patient is compliant' [2]. Even more emphatically, the AUA, and NCCN guidelines offer surveillance as the 'preferred option' $[8,9]$.

For CS1 NSGCT, options include active surveillance, retroperitoneal lymph node dissection (RPLND), or chemotherapy (typically bleomycin, etoposide and cisplatin x 1 or 2 cycles). The EAU guidelines are less direct here [2]. They suggest offering surveillance or a 
risk-adapted approach with patients in the higher-risk group being offered chemotherapy or surveillance. Primary nerve sparing RPLND is also an option for select patients. The AUA and NCCN guidelines recommend surveillance for CS1A patients while RPLND or chemotherapy are alternatives for patients who decline surveillance. For CS1B patients, they suggest shared decision making between surveillance, RPLND and chemotherapy $[8,9]$.

The Canadian guidelines recommend surveillance as the management of choice for all risk groups, in both seminoma and NSGCT. This is in line with the Princess Margaret Cancer Centre non-risk adapted approach, if the patient is willing and able to adhere to a surveillance program [10,11].

The differing opinions regarding risk adapted or non-risk adapted treatment is the main controversial feature in the management of CS1.

\section{Prognostic factors}

Certain factors have been identified as predictors of relapse for CS1 disease and these form the basis for a risk-adapted approach.

For seminoma, primary tumor size and rete testis invasion were initially identified as being associated with relapse [12]. Later studies found tumor size as the most valuable prognostic factor [13]. In a Canadian-Danish surveillance cohort of seminoma patients, Chung et al., demonstrated a continual increase in relapse risk with every centimeter increase in tumor size, ranging from $9 \%$ in a $1 \mathrm{~cm}$ tumor to $26 \%$ in an $8 \mathrm{~cm}$ tumor [14]. While a size cut-off of $>4 \mathrm{~cm}$ as 'high-risk' is consistently quoted, evidence supporting this specific cut-off is lacking. A recent systematic review by the European Testicular Cancer Guidelines Panel concluded that "the evidence on the prognostic value of size and rete testis invasion has significant limitations" and cautions against routine use in clinical practice [15].

For NSGCT, there are more consistent prognostic features. The presence of lymphovascular invasion (LVI) in the primary tumor and increasing predominance of the embryonal carcinoma component have been shown to associate with relapse risk (up to 50\%) $[16,17]$. The exact percentage of embryonal carcinoma that confers the increased risk remains unclear. Some argue the mere presence of embryonal carcinoma, others have used $>50 \%$ component, while the Princess Margaret group suggest only pure (or 100\%) embryonal carcinoma as a risk-factor given the interrater variability in assigning percent tumour involvement [11]. Because of disagreement over the embryonal component, LVI is the predominant factor behind risk-adapted guidelines. LVI upstages NSGCT from pT1 to pT2 and overall stage 1A to stage $1 \mathrm{~B}$. The largest series to date evaluating surveillance for CS1 NSGCT is a population-based cohort study from Denmark of 1226 patients [18]. They report that the presence of embryonal carcinoma (HR 3.85, 95\% CI 2.03 to 7.32), and LVI (HR 2.20, $95 \%$ CI 1.64 to 2.99 ) were significantly associated with relapse-free survival.

\section{Adjuvant therapy with chemotherapy or radiotherapy}

Professor Robert Huddart from the Royal Marsden in London discussed the advantages of adjuvant treatment and the settings in which he felt it appropriate. 
The use of adjuvant treatment is mainly reserved for patients deemed at high risk of relapse and treated in centers which advocated a risk-adapted approach. Such an approach is popular in the UK. The argument for adjuvant treatment focuses on the risks associated with noncompliance to surveillance, the intensive monitoring and cost of surveillance, the ongoing psychological stress and the resultant intensive treatment if a relapse occurs. Ernst et al., demonstrated a $22 \%$ noncompliance rate with clinic visits and a $36 \%$ noncompliance rate with imaging for patients undergoing surveillance for NSGCT across 7 Canadian centers [19]. Not surprisingly, the compliance rates were highest at the centers with less frequent visits in their protocols. Furthermore, even at dedicated cancer centres, there was a 30\% nonadherence rate to surveillance guidelines by physicians which can result in inappropriate imaging, overtreatment and related morbidity [20].

Traditionally radiotherapy had been the adjuvant treatment of choice for CS1 seminoma with studies demonstrating a reduction in relapse risk from $15 \%$ to $4 \%$ [21]. However, radiotherapy is associated with long-term consequences such as cardiovascular disease [22] and second malignancy [23], and as a result its use in CSI seminoma has declined rapidly [24].

The use of carboplatin is popular in the UK. Adjuvant chemotherapy with single agent carboplatin for either 1 or 2 cycles has been shown to reduce the risk of relapse. The phase 3 British Medical Research Council (MRC) TE19 trial demonstrated that one cycle of carboplatin was non-inferior to radiotherapy (5.3\% vs. $4 \%$ relapse) and without the long-term secondary malignancy risk [25]. In contrast, the SWENOTECA group, which may represent a more real-world experience, reported less impressive absolute risk reductions associated with carboplatin [26]. In patients with no risk factors, the relapse risk in carboplatin-treated patients compared to surveillance patients was minimally different at $2.2 \%$ vs. $4 \%$. Among patients with either one or both risk factors, the difference in relapse risk between carboplatin-treated patients (9.1-10.4\%) and surveillance (15.5\%) was larger but still much smaller than noted in the MRC TE19 trial. Aparicio et al., reported the effective use of two courses of carboplatin in patients with seminoma and high-risk features (size $>4 \mathrm{~cm}$ and rete testis invasion) [27]. Chau et al, echoed this with excellent results with the use of a single course of carboplatin with a 5-year relapse-free rate of 95\% [28].

NSGCT is less radiosensitive so the choice of adjuvant treatment lies between chemotherapy and RPLND. Cullen et al demonstrated the effectiveness of adjuvant BEP x2 in reducing the relapse rate of NSGCT from $\sim 30 \%$ to $<5 \%$ [29]. Since then, one cycle of BEP has been shown to be equally effective in reducing the relapse rate while lowering the cumulative effect of chemotherapy toxicity $[16,30]$. The recently published " $1: 1: 1$ trial" of BEP x 1 showed similar outcomes to BEP x 2 with a 2 -year recurrence free survival of $97 \%$ and a 2-year overall survival of $99 \%$ [31]. Albers et al demonstrated the benefit of one cycle of BEP over a primary RPLND in reducing the number of relapses (2 vs 13) for NSGCT [32]. 


\section{Adjuvant therapy with RPLND}

Professor Peter Albers discussed the role for primary RPLND in CS1.

Currently there is no role for RPLND in CS1 seminoma which is reflected in all the guidelines. There are two ongoing phase 2 trials evaluating primary RPLND in the setting of CSI seminoma relapse or CSIIA/B seminoma [32,33]. However, even if these trials show RPLND is safe and effective in the setting of known retroperitoneal disease it may be hard to accept as adjuvant therapy in CSI seminoma given the low risk of relapse and the lack of strong prognostic factors.

There is however a role for primary RPLND in CS1 NSGCT and it is offered by many centres. The benefit of a primary RPLND is the lack of long-term toxicity and accurate staging. Modern primary RPLND has been associated with relapse rates ranging from 0 $20 \%$, depending on pathological stage [32,34] Long-term complications can include loss of ejaculation, ventral hernia, and bowel obstruction although these are rare if performed in centres of excellence.

The advent of robotic RPLND has the potential to make this option more attractive. Robotic RPLND has been demonstrated to be feasible and safe in small series [35]. To date, 16 series of robotic RPLND have been published, with the majority reporting primary RPLND. The two largest series of primary robotic RPLND have shown 2-4 year recurrence rates similar to that of open RPLND, at 3-9\%, when adjuvant chemotherapy is given to node positive patients. Meanwhile, complication rates appear low at $6.4 \%$ overall and $1.7 \%$ Clavien Dindo $\geq 3$ [35,36] and similar to open series [37]. The Canadian Workshop felt we must exercise caution in adopting robotic RPLND until more is learned and emphasized that if robotic RPLND is to be performed it should be at expert centres with expert surgeons. Whether open or robotic, the challenge remains in selecting the appropriate patients who would benefit from surgery. Complex surgery such as RPLND needs to be performed in cancer centers by high-volume surgeons as this has been shown to be associated with less morbidity, blood loss, length of stay and fewer recurrences $[38,39]$.

\section{Surveillance}

Dr. Christian Kollmannsberger reviewed rationale and data supporting modern-era surveillance.

Surveillance has become the foremost approach for men with CS1 disease. Overall surveillance avoids treatment beyond orchiectomy in 50-75\% of patients. Based on data from the National Cancer Data Base, surveillance is the most commonly used management option for CS1 in the United States [40].

While many centres offer surveillance regardless of risk factors, a so-called, 'nonrisk-adapted' approach, debate still exists for whether surveillance is appropriate for patients with risk factors.

\section{Seminoma}

For seminoma, the argument for non-risk-adapted surveillance is most cogent. Robust prognostic markers do not exist and even in patients deemed high-risk (larger tumor size) the 
risk of relapse is only 20-25\%. Kollmannsberger reported a large review of CS1 patients from Canada, USA and Europe on surveillance - 13\% of CS1 seminomas relapsed [3]. Median time to relapse was 14 months with most (92\%) relapses occurring within 3 years. The majority of relapsed patients received cisplatin-based combination chemotherapy $(61 \%)$ while $32 \%$ underwent radiation. After a median follow-up of 52 months, no patients died of disease and only one died of treatment-related complications.

Cummins et al, assessed the treatment burden in patients who relapse on CS1 seminoma surveillance [41]. They noted a similar $13 \%$ relapse rate with the majority $82 \%$ being confined to the retroperitoneum. The disease-specific mortality was $1.3 \%$. They explored morbidity of treating relapse and measured in "treatment units" where one unit represented one cycle of chemotherapy or one course of radiation. They observed an average of 0.46 treatment units per patient or 3.45 treatment units per relapsing patient. This can be compared to a hypothetical group of CSI seminomas treated with adjuvant radiotherapy or carboplatin, where each patient would have received 1 treatment unit with only a $\sim 4 \%$ relapse risk. Thus, overall, the morbidity of surveillance is less, however, for the individual relapsing patient, the morbidity of relapse therapy may be more than if an adjuvant treatment had been chosen up-front.

However, this morbidity equation is dependent on relapse therapy chosen and also whether it is examined on an individual patient level as opposed to a population level. For example, Leung et al., reviewed the Princess Margaret experience with CSI seminoma surveillance and in contrast to other series, $78 \%$ of relapses were treated with radiation therapy, and only $12 \%$ received chemotherapy [20]. In this case, the majority of relapsing patients would have had similar treatment burden to patients choosing adjuvant therapy up front.

\section{NSGCT}

For NSGCT, the debate is more challenging for the CS1B patients where relapse risk is 40$50 \%$. However, arguments in favour of surveillance include: a) half of patients avoid any treatment beyond orchiectomy; b) the total burden of chemotherapy for the whole surveillance cohort (including those that relapse) is the same as a strategy where all receive adjuvant chemotherapy; c) relapses after adjuvant BEP may harbour worse disease biology; d) concerns about loss to follow-up are likely less prevalent or problematic than some studies suggest; and e) concerns about morbidity associated with radiation exposure of surveillance imaging are unsubstantiated. These arguments have been reviewed elsewhere in more detail [42].

In the same Kollmannsberger paper - 19\% of CS1 NSGCT patients relapsed on surveillance [40]. For all relapsing patients, median time to relapse was 6 months (4 months for LVI-positive vs. 8 months for LVI-negative). Only $1 \%$ relapsed after 3 years. Five-year disease-specific survival was $99.4 \%$. In this series capturing a multinational surveillance cohort, treatment for relapse consisted of cisplatin-based chemotherapy in $90 \%$ and primary RPLND in only 9\% (most of which were from Princess Margaret). 
Similar to seminoma, it could be argued that relapsing patients have a higher treatment burden than if they had chosen adjuvant therapy up-front. However, this burden also depends on how relapses are managed. Hamilton et al., recently reported a $28 \%$ relapse rate following a non-risk adapted approach to CS1 NSGCT surveillance [43]. The majority $(66 \%)$ relapsed within the retroperitoneum and in this series the use of RPLND as initial treatment for relapse was much higher at $38 \%$, with $73 \%$ of these patients not requiring any chemotherapy after RPLND. In their modelling exercise, a theoretical cohort of 100 highrisk (i.e. CS1B) patients treated with surveillance and salvaged preferentially with RPLND when appropriate had similar treatment burden to a group treated with adjuvant BEPx1.

\section{What is currently happening in Canada?}

Professor Christopher Booth from Queen's University presented a large population based database from the single payer system in Ontario.

Leveridge et al., recently reported the temporal trends in the management of testis cancer in Ontario [44]. Since 2000, there has been a substantial de-escalation of treatment mainly due to the adoption of surveillance as opposed to radiation treatment of CS1 seminoma. In the last year of their follow-up, $84 \%$ of all newly diagnosed seminomas (allstages) were managed with surveillance, while $57 \%$ of NSGCTs were managed with surveillance. Over the same time period the long-term survival outcomes have remained excellent: 10-year overall survival for all stages was $96 \%$ and the cancer-specific survival was $98 \%$.

The benefit of reporting population based data is a reflection of real world practice, outside of a clinical trial setting and incorporating all providers as opposed to single or multicentre of excellence studies. In this Ontario based report, $72 \%$ of patients underwent their orchiectomy in a community hospital.

The Genitourinary Cancer Disease Site Group of the Cancer Care Ontario Program produced two systematic reviews for the management of CS1 testis cancer- for both seminoma and NSGCT, surveillance is recommended for all patients [45,46]. Likewise, the previous 2010 Canadian Consensus guidelines for the management of testicular cancer support surveillance for all risk groups in patients willing to adhere to protocol [9].

\section{Future}

The goal for the future is to maintain excellent oncological outcomes while minimizing potential morbidity of treatment. The challenge remains to identify the patients at higher risk of relapse and manage them with the least associated treatment related morbidity. Prognostic serum biomarkers such as miRNA371, which is expressed in $>90 \%$ of GCT, may play a role in the choice of adjuvant therapy vs. surveillance [47]. To date, however, the ability of miRNA 371 post-orchiectomy in CS1 patients to predict future relapse remains totally unknown [48].

Given there are standardized surveillance protocols in place, surveillance for CS1 lends itself favorably towards telemedicine or virtual clinic innovations. Virtual clinics may maintain high levels of adherence to follow up schedules as it minimizes time away from 
work and daily activities for young patients; they also expand the reach of centres of excellence allowing patients in more remote locations to still receive care by a high-volume provider. A randomized trial of virtual clinic surveillance vs. standard in-person care for CS1 patients is ongoing [49]. This should provide some level 1 evidence about the feasibility, safety and satisfaction of virtual care for testis cancer CSI patients.

Finally, from a Canadian perspective, there has been a discussion regarding the role for regionalization of testis cancer. If this is to be adopted on a population basis, surveillance is a safe starting point for CS1 disease and a standardized regional protocol could be developed to follow patients with CS1 locally.

\section{Conclusions}

The majority of patients with testis cancer present with CS1 disease and, regardless of treatment approach, their outcomes are excellent. The challenge lies in a shared decision process where discussion and choice of management options extends beyond the simple risk of relapse but include the long-term toxicities of adjuvant treatments that may follow given the favorable cancer-specific survival. 


\section{References}

1. Canadian Cancer Statistics. A 2018 special report on cancer incidence by stage. www.cancer.ca (Accessed $18^{\text {th }}$ May 2020)

2. Albers P, Albrecht W, Algaba F, et al. Guidelines on Testicular Cancer: 2015 Update. Eur Urol. 2015 Dec;68(6):1054-68.

3. Kollmannsberger C, Tandstad T, Bedard PL, et al. Patterns of relapse in patients with clinical stage I testicular cancer managed with active surveillance. J Clin Oncol. 2015 Jan 1;33(1):51-7.

4. de Wit R, Bosl GJ. Optimal management of clinical stage I testis cancer: one size does not fit all. J Clin Oncol. 2013 Oct 1;31(28):3477-9.

5. Tandstad T, Cohn-Cedermark G. Primum Non Nocere: What Hurts in Clinical Stage I Testicular Cancer? J Clin Oncol. 2015 Jul 10;33(20):2318-9.

6. Trama A, Foschi R, Larrañaga N, et al. Survival of male genital cancers (prostate, testis and penis) in Europe 1999-2007: Results from the EUROCARE-5 study. Eur J Cancer. 2015 Oct;51(15):2206-2216.

7. Hamilton RJ, Jewett MAS, Warde P, et al. Optimal Management of High-risk Stage I Nonseminomatous Germ Cell Tumor: Surveillance is the Preferred Option. Eur Urol Focus. 2019;5(5):702-703.

8. Stephenson A, Eggener SE, Bass EB, et al. Diagnosis and Treatment of Early Stage Testicular Cancer: AUA Guideline. J Urol. 2019 Aug;202(2):272-281.

9. Gilligan T, Lin DW, Aggarwal R, et al. Testicular Cancer, Version 1.2019, National Comprehensive Care Network. 2019.

10. Wood L, Kollmannsberger C, Jewett M, et al. Canadian consensus guidelines for the management of testicular germ cell cancer. Can Urol Assoc J. 2010 Apr;4(2):e19-38

11. Sturgeon JF, Moore MJ, Kakiashvili DM, et al. Non-risk-adapted surveillance in clinical stage I nonseminomatous germ cell tumors: the Princess Margaret Hospital's experience. Eur Urol. 2011 Apr;59(4):556-62.

12. Warde P, Specht L, Horwich A, et al. Prognostic factors for relapse in stage I seminoma managed by surveillance: a pooled analysis. J Clin Oncol. 2002 Nov $15 ; 20(22): 4448-52$.

13. Zengerling F, Kunath F, Jensen K, et al. Prognostic factors for tumor recurrence in patients with clinical stage I seminoma undergoing surveillance-A systematic review. Urol Oncol. 2018 Oct;36(10):448-458.

14. Chung P, Daugaard G, Tyldesley S, et al. Evaluation of a prognostic model for risk of relapse in stage I seminoma surveillance. Cancer Med. 2015 Jan;4(1):155-60.

15. Boormans JL, Mayor de Castro J, Marconi L, et al.

Testicular Tumour Size and Rete Testis Invasion as Prognostic Factors for the Risk of Relapse of Clinical Stage I Seminoma Testis Patients Under Surveillance: a Systematic Review by the Testicular Cancer Guidelines Panel. Eur Urol. 2017 Nov 20. pii: S0302-2838(17)30826-6.

16. Tandstad T, Dahl O, Cohn-Cedermark G, et al. Risk-adapted treatment in clinical stage I nonseminomatous germ cell testicular cancer: the SWENOTECA management program. J Clin Oncol. 2009 May 1;27(13):2122-8.

17. Avulova S, Allen C, Morgans A, et al. Decision analysis defining optimal management of clinical stage 1 high-risk nonseminomatous germ cell testicular cancer with lymphovascular invasion. Urol Oncol. 2018 Jul;36(7):342.e1-342.e6. 
18. Daugaard G, Gundgaard MG, Mortensen MS, et al. Surveillance for stage I nonseminoma testicular cancer: outcomes and long-term follow-up in a populationbased cohort. J Clin Oncol. 2014 Dec 1;32(34):3817-23.

19. Ernst DS, Brasher P, Venner PM, et al. Compliance and outcome of patients with stage 1 non-seminomatous germ cell tumors (NSGCT) managed with surveillance programs in seven Canadian centres. Can J Urol. 2005 Apr;12(2):2575-80.

20. Wymer KM, Pearce SM, Harris KT, et al. Adherence to National Comprehensive Cancer Network $®$ Guidelines for Testicular C ancer. J Urol. 2017 Mar;197(3 Pt 1):684-689.

21. Leung E, Warde P, Jewett M, et al. Treatment burden in stage I seminoma: a comparison of surveillance and adjuvant radiation therapy. BJU Int. 2013 Dec;112(8):1088-95.

22. Fung C, Fossa SD, Williams A, et al. Long-term Morbidity of Testicular Cancer Treatment. Urol Clin North Am. 2015 Aug;42(3):393-408.

23. Travis LB, Fosså SD, Schonfeld SJ, et al. Second cancers among 40,576 testicular cancer patients: focus on long-term survivors. J Natl Cancer Inst. 2005 Sep 21;97(18):1354-65.

24. Matulewicz RS, Oberlin DT, Sheinfeld J, et al. The Evolving Management of Patients With Clinical Stage I Seminoma. Urology. 2016 Dec;98:113-119.

25. Oliver RT, Mead GM, Rustin GJ, et al. Randomized trial of carboplatin versus radiotherapy for stage I seminoma: mature results on relapse and contralateral testis cancer rates in MRC TE19/EORTC 30982 study (ISRCTN27163214).

J Clin Oncol. 2011 Mar 10;29(8):957-62

26. Tandstad T, Ståhl O, Dahl O, et al. Treatment of stage I seminoma, with one course of adjuvant carboplatin or surveillance, riskadapted recommendations implementing patient autonomy: a report from the Swedish and Norwegian Testicular Cancer Group (SWENOTECA). Ann Oncol. 2016 Jul;27(7):1299-304.

27. Aparicio J, Germa JR, Garcia del Muro X, et al. Risk adapted management for patients with clinical stage I seminoma: the Second Spanish Germ Cell Cancer Cooperative Group study. J Clin Oncol. 2005 Dec 1;23(34):8717-23

28. Chau C, Cathomas R, Wheater M, et al. Treatment outcome and patterns of relapse following adjuvant carboplatin for stage I testicular seminomatous germ-cell tumour: results from a 17-year UK experience. Ann Oncol. 2015 Sep;26(9):1865-70.

29. Cullen MH, Stenning SP, Parkinson MC, et al. Short-course adjuvant chemotherapy in high-risk stage I nonseminomatous germ cell tumors of the testis: a Medical Research Council report. J Clin Oncol. 1996 Apr;14(4):1106-13.

30. Gilbert DC, Norman AR, Nicholl J, et al. Treating stage I nonseminomatous germ cell tumours with a single cycle of chemotherapy. BJU Int. 2006 Jul;98(1):67-9.

31. Cullen M, Huddart R, Joffe J, et al. The 111 Study: A Single-arm, Phase 3 Trial Evaluating One Cycle of Bleomycin, Etoposide, and Cisplatin as Adjuvant Chemotherapy in High-risk, Stage 1 Nonseminomatous or Combined Germ Cell Tumours of the Testis. Eur Urol. 2020 Mar;77(3):344-351.

32. Albers P, Siener R, Krege S, et all.

Randomized phase III trial comparing retroperitoneal lymph node dissection with one course of bleomycin and etoposide plus cisplatin chemotherapy in the adjuvant treatment of clinical stage I Nonseminomatous testicular germ cell tumor 
s: AUO trial AH 01/94 by the German Testicular Cancer Study Group. J Clin Oncol. 2008 Jun 20;26(18):2966-72.

33. https://clinicaltrials.gov/ct2/show/NCT02537548 (Accessed 18th May 2020)

34. Stephenson AJ, Bosl GJ, Bajorin DF, et al. Retroperitoneal lymph node dissection in patients with low stage testicular cancer with embryonal carcinoma predominance and/or lymphovascular invasion. J Urol. 2005 Aug;174(2):557-60; discussion 560

35. Pearce SM, Golan S, Gorin MA, et al. Safety and Early Oncologic Effectiveness of Primary Robotic Retroperitoneal Lymph Node Dissection for Nonseminomatous Germ Cell Testicular Cancer. Eur Urol. 2017 Mar;71(3):476-482.

36. Rocco NR, Stroup SP, Abdul-Muhsin HM, et al. Primary robotic RLPND for nonseminomatous germ cell testicular cancer: a two-center analysis of intermediate oncologic and safety outcomes. World J Urol. 2020 Apr;38(4):859-867.

37. Heidenreich A, Albers P, Hartmann M, et al..

Complications of primary nerve sparing retroperitoneal lymph node dissection for clin ical stage I nonseminomatous germ cell tumors of the testis: experience of the German Testicular Cancer Study Group. J Urol. 2003 May;169(5):1710-4.

38. Capitanio U, Jeldres C, Perrotte P, et al. Populationbased study of perioperative mortality after retroperitoneal lymphadenectomy for non seminomatous testicular germ cell tumors. Urology. 2009 Aug;74(2):373-7.

39. Fléchon A, Tavernier E, Boyle H, et al. Long-term oncological outcome after post chemotherapy retroperitoneal lymph node dissectionin men with metastatic nonsemin omatous germ cell tumour. BJU Int. 2010 Sep;106(6):779-85.

40. Jeldres C, Nichols C, Pham K, et al: United States trends in patterns of care in clinical stage I testicular cancer: Results from the National Cancer Database (1998-2011). J Clin Oncol 32:5s,2014 (suppl; abstr 4519)

41. Cummins S, Yau T, Huddart R, Dearnaley D, et al. Surveillance in stage I seminoma patients: a long-term assessment. Eur Urol. 2010 Apr;57(4):673-8.

42. Nason GJ, Leao R, Hamilton RJ. Active surveillance in stage 1 disease: standard of care independent of risk factors? Curr Opin Urol. 2020 Mar;30(2):245-250.

43. Hamilton RJ, Nayan M, Anson-Cartwright L, et al. Treatment of Relapse of Clinical Stage I Nonseminomatous Germ Cell Tumors on Surveillance. J Clin Oncol. 2019 Aug 1;37(22):1919-1926.

44. Leveridge MJ, Siemens DR, Brennan K, et al. Temporal trends in management and outcomes of testicular cancer: A population-based study. Cancer. $2018 \mathrm{Jul}$ 1;124(13):2724-2732.

45. Hotte SJ, Mayhew LA, Jewett M, et al. Management of stage I non-seminomatous testicular cancer: a systematic review and meta-analysis. Clin Oncol (R Coll Radiol). 2010 Feb;22(1):17-26.

46. Chung P, Mayhew LA, Warde P, et al. Management of stage I seminomatous testicular cancer: a systematic review. Clin Oncol (R Coll Radiol). 2010 Feb;22(1):616.

47. Dieckmann KP, Radtke A, Spiekermann M, et al. Serum Levels of MicroRNA miR371a-3p: A Sensitive and Specific New Biomarker for Germ Cell Tumours. Eur Urol. 2017 Feb;71(2):213-220.

48. 48.Leão R, Ahmad AE, Hamilton RJ. Testicular Cancer Biomarkers: A Role for Precision Medicine in Testicular Cancer. Clin Genitourin Cancer. 2019 Feb;17(1):e176-e183. https://clinicaltrials.gov/ct2/show/NCT03360994 (Accessed 18th May 2020) 